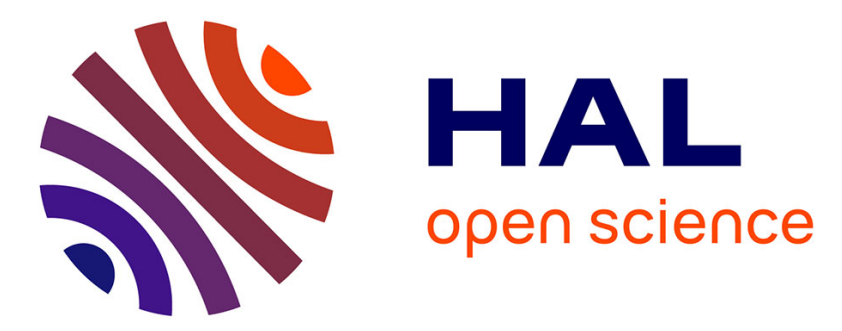

\title{
Possibility of superconducting precursor effects in quasi-one-dimensional organic conductors : theory and experiments
}

\author{
H.J. Schulz, D. Jérome, A. Mazaud, M. Ribault, K. Bechgaard
}

\section{- To cite this version:}

H.J. Schulz, D. Jérome, A. Mazaud, M. Ribault, K. Bechgaard. Possibility of superconducting precursor effects in quasi-one-dimensional organic conductors : theory and experiments. Journal de Physique, 1981, 42 (7), pp.991-1002. 10.1051/jphys:01981004207099100 . jpa-00209091

\section{HAL Id: jpa-00209091 https://hal.science/jpa-00209091}

Submitted on 1 Jan 1981

HAL is a multi-disciplinary open access archive for the deposit and dissemination of scientific research documents, whether they are published or not. The documents may come from teaching and research institutions in France or abroad, or from public or private research centers.
L'archive ouverte pluridisciplinaire HAL, est destinée au dépôt et à la diffusion de documents scientifiques de niveau recherche, publiés ou non, émanant des établissements d'enseignement et de recherche français ou étrangers, des laboratoires publics ou privés. 


\title{
Possibility of superconducting precursor effects in quasi-one-dimensional organic conductors : theory and experiments
}

\author{
H. J. Schulz (*), D. Jérome, A. Mazaud, M. Ribault \\ Laboratoire de Physique des Solides, Université Paris-Sud, 91405 Orsay, France
}

and $\mathrm{K}$. Bechgaard

H.C. Oersted Institute, Universitetsparken 5, D.K. 2100 Copenhagen, Denmark.

(Reçu le 27 novembre 1980, accepté le 17 mars 1981)

\begin{abstract}
Résumé. - Nous présentons des mesures de la conductivité et de son anisotropie effectuées sur le conducteur organique quasi unidimensionnel (TMTSF) ${ }_{2} \mathrm{PF}_{6}$ sous $12 \mathrm{kbar}$ entre $200 \mathrm{~K}$ et la transition supraconductrice. Il apparaît difficile d'admettre comme interprétation des résultats un mécanisme de conduction en terme de conduction de particules individuelles. Une telle interprétation conduirait à une très grande valeur du libre parcours moyen électronique d'environ $3000 \AA ̊$ à la température de l'hélium liquide. Au lieu de la précédente interprétation nous proposons une explication en terme de paraconduction induite par les fluctuations supraconductrices. Nous présentons un calcul de cette paraconductivité effectué au moyen de la théorie de Landau-Ginzburg dépendant du temps. L'accord quantitatif satisfaisant entre le modèle et les résultats expérimentaux suggère bien que les fluctuations supraconductrices jouent un rôle important jusqu'à $40 \mathrm{~K}$ environ. Le même modèle indique en outre que la susceptibilité n'est pas augmentée par les fluctuations de façon significative.
\end{abstract}

\begin{abstract}
We report an investigation of the temperature dependence of the conductivity and conductivity anisotropy in the single-chain quasi-one-dimensional organic conductor (TMTSF) ${ }_{2} \mathrm{PF}_{6}$ under 12 kbar between $200 \mathrm{~K}$ and the superconducting transition temperature. An interpretation of the data in terms of a single-particle conduction mechanism would lead to a huge mean free path of about $3000 \AA$ at helium temperature which seems rather hard to accept. Instead, we propose an interpretation based on superconducting fluctuations induced paraconductivity. The calculation is performed with a time dependent Landau-Ginzburg theory. The satisfying quantitative agreement between the theoretical model and the experimental data points out the dominant role of superconducting fluctuations in this conductor up to about $40 \mathrm{~K}$. The theory shows that unlike conductivity the susceptibility is not significantly enhanced by the fluctuations in the same temperature domain.
\end{abstract}

1. Introduction. - Recent transport properties studies of the quasi-one-dimensional organic conductors TMTSF-DMTCNQ and (TMTSF) ${ }_{2} \mathrm{PF}_{6}$ have shown that the conducting state stabilized at low temperature under a pressure larger than 10 kbar exhibits several interesting features $[1,2]$ :

i) At helium temperature the longitudinal conductivity exceeds the value of $10^{5}(\Omega \mathrm{cm})^{-1}$ which is roughly an order of magnitude larger than the figure obtained in other conducting materials.

In most organic conductors a conductivity of $\approx 10^{4}(\Omega \mathrm{cm})^{-1}$ seems to be an upper limit ; for example, this is the value observed at $60 \mathrm{~K}$ in TTF-TCNQ [3] at $40 \mathrm{~K}$ in TSF-TCNQ [4], and at helium temperature

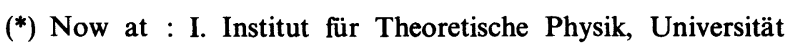
Hamburg, 2000 Hamburg 36, West Germany. in imperfect HMTSF-TCNQ crystals under pressure above 4 kbar [5].

ii) When compared to regular 3-D metals the resistivity of (TMTSF) ${ }_{2} \mathrm{PF}_{6}$ [6] does exhibit a particular feature, namely a very strong temperature dependence in the helium temperature range, whereas in the same temperature domain the resistance of ordinary 3-D metals tends to saturate towards a residual resistance value limited by lattice imperfections or impurities.

iii) A transition towards a superconducting state is observed at $0.9 \mathrm{~K}$ in (TMTSF) ${ }_{2} \mathrm{PF}_{6}$ under $12 \mathrm{kbar}$. It has been characterized by a drop of the sample resistance to a zero value [7] and the existence of diamagnetic currents in the bulk superconductor below $0.9 \mathrm{~K}$ [8]. Very large magnetoresistance effects have been reported well above the superconducting 
transition at $12 \mathrm{kbar}$ [7] and also at $20 \mathrm{~K}$ under ambient pressure [6].

The purpose of the present study is threefold, first a thorough investigation of the (TMTSF) ${ }_{2} \mathrm{PF}_{6}$ longitudinal and transverse resistivities in the whole conducting domain of temperatures at $12 \mathrm{kbar}$. Secondly, a discussion which shows that transport properties at low temperature cannot be interpreted in terms of regular metallic or semimetallic behaviours and thirdly the presentation of a simple theoretical model for the superconductivity induced paraconduction, in a quasi-one-dimensional conductor above $T_{\mathrm{c}}$.

We shall also comment in a discussion section on the relation between the paraconductivity theoretical model and the experimental data. The final section will deal with the problem of the stability of the superconducting state versus the Peierls state in an organic conductor.

2. Experimental results. - The resistance of (TMTSF) ${ }_{2} \mathrm{PF}_{6}$ single crystals obtained from ultrapure constituents by an electrochemical technique [6] has been measured either by usual low frequency techniques $(v<100 \mathrm{~Hz})$ or by $\mathrm{DC}$ techniques. Typical crystals sizes were $4 \times 0.2 \times 0.1 \mathrm{~mm}^{3}$ and some crystals were large enough to allow a direct measurement of the transverse resistivity. For the case of one crystal we have been able to performe simultaneously the measurement of both conductivity components on the same specimen using a 6-contact arrangement, whereas for all other cases the two components of the conductivity were measured independently on different crystals. Contacts between thin gold wires and the sample were made with either silver or gold dags. Contact resistances were under $10 \Omega$ and great care has been taken in the experiments as to keep unnested voltages at a level always smaller than $10 \%$ of the nested voltages.

Two different pressure equipments have been used for this experiment : a steel pressure vessel for nonmagnetic measurements down to $1.2 \mathrm{~K}$ and at lower temperatures a clamped copper-beryllium vessel cooled by a ${ }^{3} \mathrm{He}-{ }^{+} \mathrm{He}$ dilution refrigerator [7]. The temperature is measured inside the pressure bomb by a copper-constantan thermocouple down to $4.2 \mathrm{~K}$ and by carbon or germanium thermometers at lower temperatures.

Room temperature transverse conductivities range from 0.1 to $0.4(\Omega \mathrm{cm})^{-1}$ in four different crystals suggesting an average value of $\sigma_{\perp}=0.25(\Omega \mathrm{cm})^{-1}$. This value is somewhat smaller than the first published DC data for the direction labelled 1 en reference [6], but we feel confident that the transverse direction chosen in the present measurements corresponds roughly to the Se-Se intermolecular direction, since a measure of $\sigma_{\perp}$ along the other perpendicular direction gave a value of only $0.03(\Omega \mathrm{cm})^{-1}$. The longitudinal conductivity was in average not quite as large as that reported earlier [6] and the room temperature ambient pressure value amounts to $\approx 400 \pm 100(\Omega \mathrm{cm})^{-1}$. Therefore, the conductivity anisotropy is about $10^{3}$ for the transverse direction investigated in this work and $\approx 10^{4}$ for the other transverse direction, which has not been studied at low temperature. Both $\perp$ and $\|$ conductivities increase under pressure at room temperature at a common rate of $\approx 23 \% \mathrm{kbar}^{-1}$ in the low pressure domain. We have succeeded several times in the measurement of $\sigma_{\perp}$ up to $12 \mathrm{kbar}$ without voltage jumps $\left(\sigma_{\perp}(12 \mathrm{kbar}) / \sigma_{\perp}(1 \mathrm{~atm})=3.3.\right)$. However, as far as the longitudinal component is concerned, small sample breakings are usually noticed above $4 \mathrm{kbar}$ or so, resulting in minor voltage jumps. But a much better stability of the measurements is reached as soon as the isopentane pressure medium freezes, namely below $210 \mathrm{~K}$ under $12 \mathrm{kbar}$.

Therefore, the measurements reported in this work are displayed only below $200 \mathrm{~K}$.

The longitudinal resistivity of (TMTSF) ${ }_{2} \mathrm{PF}_{6}$ decreases monotonously $\approx 100 \mathrm{X}$ from $200 \mathrm{~K}$ to $4.2 \mathrm{~K}$. A somewhat similar overall temperature dependence is observed for the transverse resistivity with the special features that first the ratio $\rho_{\perp}(200 \mathrm{~K}) / \rho_{\perp}$ $(4.2 \mathrm{~K})$ is larger than 200 and secondly that the drop of resistance below $50 \mathrm{~K}$ is faster for the transverse than for the longitudinal component. Figure 1 displays the temperature dependence of the longitudinal resistance below $200 \mathrm{~K}$ under $12 \mathrm{kbar}$. The temperature

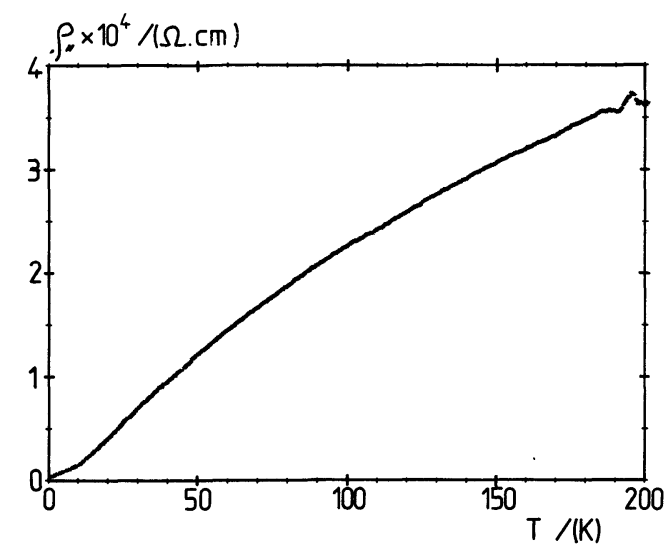

Fig. 1. - Temperature dependence of the longitudinal resistivity of (TMTSF) ${ }_{2} \mathrm{PF}_{6}$ below $200 \mathrm{~K}$ at $12 \mathrm{kbar}$ pressure.

dependence of the conduction anisotropy is shown on figures $2 a$ and $2 b$. Figure $2 a$ displays the anisotropy data derived from the measurements on the same sample, whereas figure $2 b$ presents the anisotropy obtained from the ratio of independent measurements of $\sigma_{\perp}$ and $\sigma_{\|}$on different crystals.

Figure 2 reveals that $\sigma_{\|} / \sigma_{\perp}$ shows a weakly pronounced maximum around $100 \mathrm{~K}$. A very significant decrease of $\sigma_{\|} / \sigma_{\perp}$ is observed below $50 \mathrm{~K}$, by a factor 3 roughly at helium temperature. 


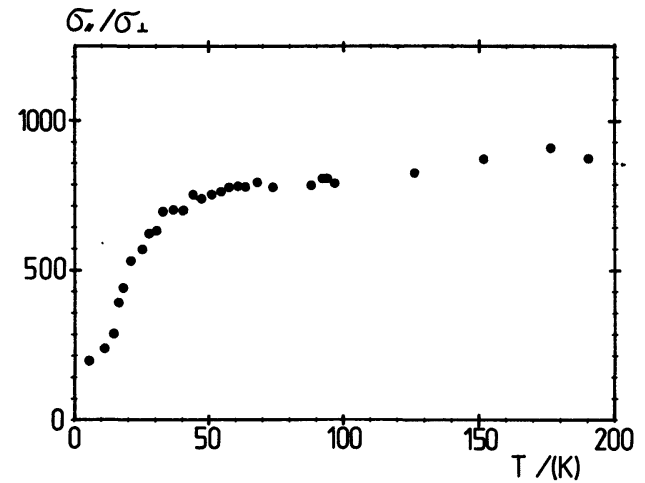

a)

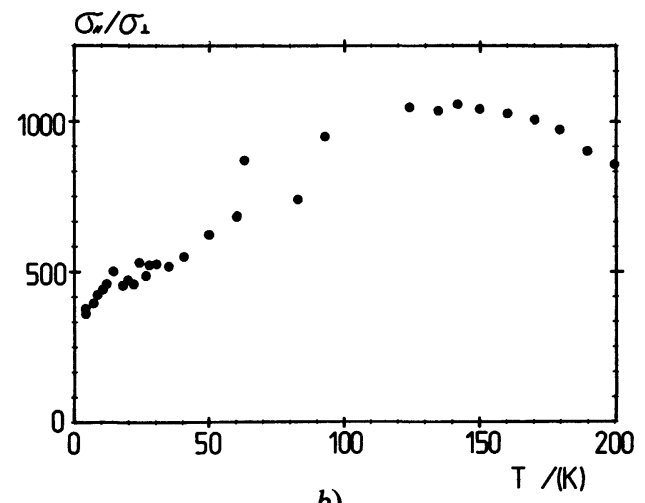

b)

Fig. 2. - Temperature dependence of the conductivity anisotropy $\sigma_{\|} / \sigma_{\perp}$ at $12 \mathrm{kbar}:(a)$ with $\sigma_{\|}$and $\sigma_{\perp}$ measured on the same sample, (b) on different samples.

Figure 3 shows the temperature dependence of the longitudinal resistance from $40 \mathrm{~K}$ down to $20 \mathrm{mK}$.

Besides weak changes of the ratio $t_{\|} / t_{\perp}$ which can be expected as either temperature or pressure are varied, $\sigma_{\|} / \sigma_{\perp}$ must be essentially $T$ and $P$ independent. The previous situation is encountered in TTFTCNQ and TSF-TCNQ [10], as pressure is increased at room temperature since the conductivity anisotropy remains practically constant up to $10 \mathrm{kbar}$. A temperature independent anisotropy is also met in TTF-TCNQ at ambient pressure between 300 and $150 \mathrm{~K}$ and practically over the entire metallic region

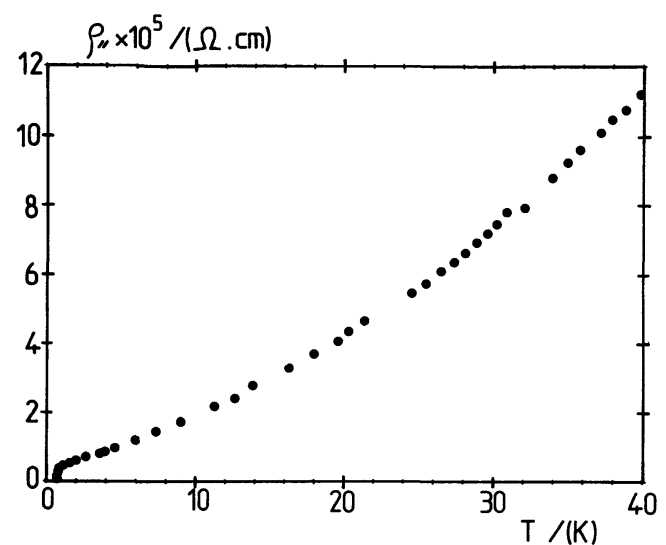

Fig. 3. - Temperature dependence of the longitudinal resistivity of (TMTSF) ${ }_{2} \mathrm{PF}_{6}$ below $40 \mathrm{~K}$ at $12 \mathrm{kbar}$ pressure. when pressure is such as to achieve longitudinal commensurability $\left(2 k_{\mathrm{F}}=b^{*} / 3\right)$ around $19 \mathrm{kbar}[11$, 12].

As far as the incommensurate metallic state of TTFTCNQ is concerned, the conductivity anisotropy is clearly temperature dependent below $150 \mathrm{~K}$; increasing by a factor 5 between 150 and $60 \mathrm{~K}$. This behaviour is the result of the CDW fluctuations contributing to the $\|$ conduction but leaving the $\perp$ conduction unaffected [13].

The situation where the anisotropy decreases towards low temperatures raises more puzzling problems.

As $\tau_{\|}$is increasing at low temperature, due to the weakening of the various single particle electron scattering processes a $1-\mathrm{D}$ band picture remains no longer valid when $t_{\perp} \approx \hbar / \tau_{\|}$. This condition can be met in a compound for which the 3-D coupling $t_{\perp}$ is large and the impurity content low enough [14] ( $\tau_{\|}$large at low temperature). Thus a diffusive to coherent transition of the transverse conduction can take place at low temperature. Moreover, a drop of the anisotropy is associated with this change over from diffusive to coherent motion since $\sigma_{\|} / \sigma_{\perp}=\Lambda_{\|} / \Lambda_{\perp}$. The ratio of mean free paths in an anisotropic conductor is $t_{\|} / t_{\perp}$. Therefore the anisotropy is expected to drop from $\left(t_{\|} / t_{\perp}\right)^{2}$ at high temperature to $t_{\|} / t_{\perp}$ at low temperature.

Why not take the above-described interpretation for the behaviour of (TMTSF) ${ }_{2} \mathrm{PF}_{6}$ ? since (i) $\sigma_{\perp} \approx 150(\Omega \mathrm{cm})^{-1}$ at $1.2 \mathrm{~K}$ (i.e. a value which could be described by a coherent band picture [15]) and (ii) $t_{\perp} \sim 4 \mathrm{meV}$ with $t_{\perp}=t_{\|} / 30$, where $t_{\|}$is derived from optical spectra or thermopower data [6] and thus the very large conductivity at low temperature could lead to $t_{\perp} \gtrsim \hbar / \tau_{\|}$.

Assuming the picture of an anisotropic 3-D conductor to be valid for (TMTSF) ${ }_{2} \mathrm{PF}_{6}$ under $12 \mathrm{kbar}$ at low temperature would, lead to the following consequences : given the electron density $n \approx 10^{22}$ charges $/ \mathrm{cm}^{3}$, the conductivity of $5 \times 10^{5}(\Omega \mathrm{cm})^{-1}$ leads to mean free paths of $\approx 2800 \AA$ (i.e. 700 intermolecular spacings), corresponding to a mobility of $2 \times 10^{3} \mathrm{~cm}^{2} /$ V.s at low temperature. Such a large mobility requires a degree of purity and perfection unlikely to be met in organic conductors. However large mobilities have been measured under very special circumstances in organic conductors, when, for example, a semimetallic state can be stabilized by pressure in disordered HMTSF-TCNQ crystals [5, 16].

Low temperature galvanomagnetic measurements [17] have shown that the carrier mobility may be fairly large, of the order of $10^{4} \mathrm{~cm}^{2} / \mathrm{V} . \mathrm{s}$ or so. Such a large value is compatible with the band structure of HMTSF-TCNQ, which is a two-chain conductor [18]. Consequently, as a result of the hybridization between neighbouring chains of different nature the 
Fermi surface undergoes a transformation between that of a 1-D conductor at high temperature and that of a 3-D anisotropic semimetal at low temperature.

Unlike 1-D Fermi surfaces, the Fermi wave vector becomes small at low temperature in a semimetallic band model $\left(\delta k_{\mathrm{F}} \sim 10^{-2} k_{\mathrm{F}}\right)$. Thus, a mean free path of $50 \AA$ or so is derived eventhough the mobility is very large [18]. (TMTSF) ${ }_{2} \mathrm{PF}_{6}$ is a single-chain conductor and as such closed Fermi surface cannot form unless $t_{\perp}$ becomes of the order of $t_{\|}$, which is obviously far from being satisfied. Moreover, the conductivity anisotropy is still $\approx 300$ at low temperature, whereas a 3-D anisotropic band model would predict only 30 (given the measured value of 1000 at room temperature).

In summary, the diffusive to coherent model does not lead to a reasonable interpretation of the transport measurements of (TMTSF $)_{2} \mathrm{PF}_{6}$ performed in this work. The experiments suggest that one must look for an other interpretation, possibly in terms of conduction being strongly influenced by collective mechanisms whenever the conductivity exceeds $10^{4}(\Omega \mathrm{cm})^{-1}$. The theory for an other interpretation is described in the forthcoming section.

3. Superconducting fluctuations in a quasi-one-dimensional metal : Ginzburg-Landau theory. - A convenient way to treat fluctuation effects in superconductors is the Ginzburg-Landau theory. Though this approach suffers from serious limitations, especially in quasi-one-dimensional conductors, as discussed later, it provides a starting point of the understanding of the fluctuation effects.

In the microscopic derivation of the coefficients of the GL theory the Cooper-pair propagator plays a central role $[19,20]$. To derive this function we use a model system of square array of parallel conducting chains with the possibility of electron tunnelling between adjacent chains. The single electron propagator is

$$
G\left(\mathbf{q}, \omega_{n}\right)=\left(i\left\{\omega_{n}+\operatorname{sign}\left(\omega_{n}\right) 1 / 2 \tau\right\}-\varepsilon(\mathbf{q})\right)^{-1}
$$

where the free-electron energy is given by :

$$
\varepsilon(\mathbf{q})=\frac{1}{2 m} q_{z}^{2}+J\left(\cos q_{x} a+\cos q_{y} a\right)-\varepsilon_{\mathrm{F}}
$$

$J / 2$ and $a$ are the transverse tunnelling integral $t_{\perp}$ and lattice constant respectively and $\tau$ is the singleelectron lifetime due to impurity scattering within a single-chain and in this section we set $\hbar=1$.

The calculations follow closely reference [19] and the free energy is given to lowest order in the Fourier components $\psi_{k}$ of the superconducting order parameter by :

$$
\begin{aligned}
F=\sum_{\mathbf{q}}\{\alpha & +\frac{q_{z}^{2}}{2 m}+\frac{J^{2}}{v_{\mathbf{F}}^{2} m} \times \\
& \left.\times\left(2-\cos q_{x} a-\cos q_{y} a\right)\right\}\left|\psi_{\mathbf{q}}\right|^{2}
\end{aligned}
$$

with

$$
\alpha=\frac{8 \pi^{2} T^{2}}{7 \zeta(3) m v_{\mathrm{F}}^{2} \chi\left((2 \pi T \tau)^{-1}\right)} \ln \frac{T}{T_{\mathrm{c}}^{0}}=\alpha^{\prime} \ln \frac{T}{T_{\mathrm{c}}^{0}}
$$

where $v_{\mathrm{F}}$ is the Fermi velocity, $T_{\mathrm{c}}^{0}$ the mean-field transition temperature and $\chi(x)$ Gorkov's $\chi$-function [19]. The effect of higher-order terms in the free energy, important in one-dimension, will be discussed below. From equation (3.1) the longitudinal and transverse temperature dependent correlation lengths are [20] :

$$
\xi_{\|}(T)=(2 m \alpha)^{-1 / 2}, \quad \xi_{\perp}(T)=\frac{J a}{v_{\mathrm{F}}(2 m \alpha)^{1 / 2}}
$$

It is interesting to note that the transverse coupling in (3.1) is proportional to $J^{2}$, i.e. to the square of the transverse electronic effective mass at Fermi level. This is a consequence of the weak interchain coupling, which may be considered as Josephson coupling, and therefore the anisotropy of the order parameter fluctuations as given by $\xi_{\|}(T) / \xi_{\perp}(T)$ is equal to the anisotropy of the electronic spectrum. A similar result has been found for weakly coupled superconducting layers [21] and for coupled Peierls chains [22]. The $J^{2}$-dependence is valid as long as the transverse coupling is smaller than the Fermi energy, i.e. as long as the Fermi surface is open in the transverse directions. On the other hand, if the Fermi surface becomes closed $\left(J \gtrsim \varepsilon_{\mathrm{F}}\right)$, the transverse coupling is linear in $J$ [23], so that the order parameter anisotropy is proportional to the square root of the anisotropy of the electronic spectrum. This case may well be described by an anisotropic effective mass of the electrons [24, 25 ]. However, in organic conductors one has typically $J / \varepsilon_{\mathrm{F}} \approx 10^{-2}[9,26]$, so that the weak coupling condition is very well satisfied.

The longitudinal and transverse supercurrents are given by :

$$
\begin{gathered}
j_{\|}=\frac{2 e}{m} \sum_{\mathbf{q}} q_{z}\left|\psi_{\mathbf{q}}\right|^{2}, \\
j_{\perp}=\frac{2 e a J^{2}}{m v_{\mathbf{F}}^{2}} \sum_{\mathbf{q}} \sin q_{x} a\left|\psi_{\mathbf{q}}\right|^{2} .
\end{gathered}
$$

For the calculation of the fluctuation conductivity, a knowledge of the dynamics of the order parameter is necessary [27]. In time-dependent Ginzburg-Landau theory $[28,29]$ this is given by the equation of motion :

$$
\frac{\pi \alpha^{\prime}}{8 T} \frac{\partial \psi_{\mathbf{k}}}{\partial t}+\frac{\partial F}{\partial \psi_{\mathbf{k}}^{*}}=0
$$

We follow now closely the method of reference [27] to calculate the contribution of the superconducting fluctuations to the conductivity. 
An approximate evaluation of the resulting integrals [23] gives :

$$
\sigma_{\|}^{\prime}=\frac{v_{\mathrm{F}}^{2}}{J^{2} a^{2}} \frac{e^{2} \alpha^{\prime}}{16}\left(\frac{m}{2 \alpha}\right)^{1 / 2}\left[1-\frac{a}{\left(a^{2}+4 \pi \xi_{\perp}^{2}\right)^{1 / 2}}\right]
$$

$$
\begin{aligned}
\sigma_{\perp}^{\prime}= & \frac{e^{2} \alpha^{\prime}}{16}\left(\frac{m}{2 \alpha}\right)^{1 / 2} \times \\
& \times\left[1-\frac{3 a}{2\left(a^{2}+4 \pi \xi_{\perp}^{2}\right)^{1 / 2}}+\frac{a^{3}}{2\left(a^{2}+4 \pi \xi_{\perp}^{2}\right)^{3 / 2}}\right]
\end{aligned}
$$

for the longitudinal and transverse conductivity, respectively. The transverse correlation length $\xi_{\perp}$ is given by (3.3).

We now consider two limiting cases :

i) near $T_{\mathrm{c}}^{0}, \alpha$ becomes sufficiently small, so that the transverse coherence length becomes larger than the lattice spacing. Then, the fluctuations are essentially three-dimensional and (3.6) reduces to :

$$
\sigma_{\|}^{3 \mathrm{D}}=\frac{v_{\mathrm{F}}^{2}}{J^{2} a^{2}} \frac{e^{2} \alpha^{\prime}}{16}\left(\frac{m}{2 \alpha}\right)^{1 / 2}, \quad \sigma_{\perp}^{3 \mathrm{D}}=\left(\frac{J a}{v_{\mathrm{F}}}\right)^{2} \sigma_{\|}^{3 \mathrm{D}} .
$$

This shows the typical $\left(T-T_{\mathrm{c}}^{0}\right)^{-1 / 2}$ divergence of a three-dimensional superconductor [27] in both the longitudinal and the transverse conductivity, however the longitudinal value is enhanced over its value in an isotropic superconductor by a factor $v_{\mathrm{F}}^{2} / J^{2} a^{2}$, which may be very large in organic conductors due to the small interchain tunnelling integral. This enhancement is due to the small transverse coherence length compared to $\xi_{\|}$which leads to a large mean square amplitude of the superconducting order parameter. The anisotropy of the paraconductivity is the same as the anisotropy of the single electron conductivity, provided that the transverse single electron conductivity is diffusive [9].

ii) At temperatures sufficiently above $T_{\mathrm{c}}^{0}, \xi_{\perp}$ becomes smaller than the interchain distance. If $\xi_{\perp}(T) \ll a$, fluctuations on adjacent chains are essentially uncorrelated and can therefore be considered as one-dimensional. The longitudinal and transverse conductivities then follow from (3.6) as :

$$
\begin{aligned}
\sigma_{\|}^{1 \mathrm{D}} & =\frac{\pi e^{2}}{16 a^{2}} \frac{\alpha^{\prime}}{(2 m)^{1 / 2} \alpha^{3 / 2}} \\
\sigma_{\perp}^{1 \mathrm{D}} & =\frac{3 \pi^{2} e^{2}}{32} \frac{J^{4}}{m v_{\mathrm{F}}^{4}} \frac{\alpha^{\prime}}{(2 m)^{1 / 2} \alpha^{5 / 2}}
\end{aligned}
$$

and the anisotropy is :

$$
\frac{\sigma_{\|}^{1 \mathrm{D}}}{\sigma_{\perp}^{1 \mathrm{D}}}=\frac{1}{3 \pi} \frac{a^{2} \xi_{\|}^{2}(T)}{\xi_{\perp}^{4}(T)}
$$

$\sigma_{\|}^{1 D}$ is the well known result for a one-dimensional superconductor [27]. A transverse supercurrent between adjacent chains requires a well developed order parameter on the two chains. As the probability for this to happen decreases with increasing temperature, for $\xi_{\perp}<a, \sigma_{\perp}^{1 \mathrm{D}}$ decreases much faster than $\sigma_{\|}^{1 \mathrm{D}}$. Correspondingly, the anisotropy of the fluctuation conductivity, equation (3.9), is much larger than the anisotropy of the single-electron conductivity, so that there may be a considerable contribution of fluctuations to the longitudinal conductivity, whereas the transverse conductivity is dominated by singleelectron conduction.

In figure 4, we show the variation of the longitudinal resistivity $\left(\sigma^{\prime-1}\right)$ with varying $\xi_{\perp}(T)$ as resulting from equation (3.6a), together with its three-dimensional (3.7) and one-dimensional (3.8a) limiting forms. The figure shows that deviations from the 3-D behaviour become important for $\xi_{\perp}(T) \lesssim a$, however the 1-D limiting form is only slowly approached with decreasing $\xi_{\perp}$.

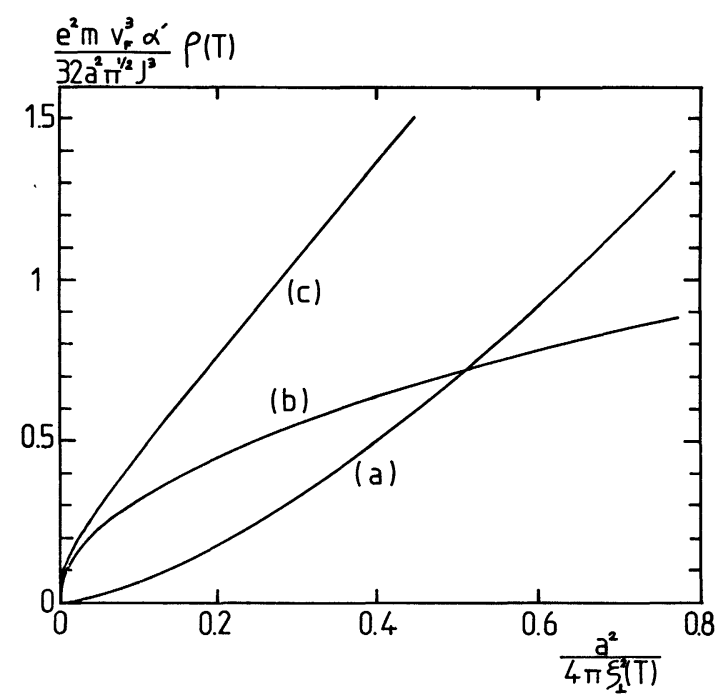

Fig. 4. - The theoretical result for the fluctuation resistivity of a quasi-one-dimensional metal : (a) strictly one-dimensional form (Eq. $(3.8 a)),(b)$ isotropic system with $\xi_{\|}=\xi_{\perp}$, and $(c)$ the general form (Eq. (3.6a)).

Another quantity of interest is the fluctuation induced diamagnetism. This effect can be calculated including a vector potential in the free energy function (3.1), as done by Turkevich and Klemm [30]. It is then straightforward to calculate the fluctuation diamagnetism, following the method of Schmid [31] with the results :

$$
\begin{aligned}
& \chi_{\perp}=-\frac{k T}{6 \pi} \frac{e^{2}}{c^{2}} \xi_{\|}(T) \frac{\xi_{\perp}(T)}{\left(\xi_{\perp}^{2}(T)+\frac{a^{2}}{4}\right)^{1 / 2}} \\
& \chi_{\|}=-\frac{k T}{6 \pi} \frac{e^{2}}{c^{2}} \frac{\xi_{\perp}^{2}(T)}{\xi_{\|}(T)}
\end{aligned}
$$


for a field applied perpendicular and parallel to the chain direction respectively. Both expressions apply in the low field limit. Equation (3.10a) shows that the fluctuation diamagnetism is not enhanced over its value in an isotropic superconductor with $\xi(T)=\xi_{\|}(T)$. This result may be understood noting that the circular Meissner currents which are at the origin of the diamagnetism have to pass between adjacent chains. Due to the weak coupling (Eq. (3.4)) the currents between chains are strongly suppressed. This effect compensates (for perpendicular field and $\left.\xi_{\perp} \gg a\right)$ the large amplitude of the fluctuations, so that for $\xi_{\perp} \gg a, \chi_{\perp}$ is the same as in an isotropic superconductor. For $\xi_{\perp} \lesssim a$, the interchain currents are further suppressed, so that $\chi_{\perp}$ is reduced. In a parallel field, the Meissner currents pass only between the chains and never along the chains. Therefore $\chi_{\|}$is always smaller than $\chi_{\perp}$.

Up to now we have considered the fluctuation effects only in lowest order, so that the quadratic expansion (3.1) is sufficient. However, it is well known that in one-dimensional systems fluctuation effects are strong and therefore the lowest order terms are not sufficient and a fourth order term :

$$
F_{4}=\frac{b}{V} \sum_{\mathbf{k p q}} \psi_{\mathbf{k}}^{*} \psi_{\mathbf{p}}^{*} \psi_{\mathbf{q}}, \psi_{\mathbf{k}+\mathbf{p}-\mathbf{q}}
$$

with :

$$
b=\frac{\alpha^{\prime}}{n \chi\left((2 \pi T \tau)^{-1}\right)}
$$

has to be included, where $n$ is the total electron density.

The width of the temperature region where strong fluctuations appear is given by the Ginzburg critical region [32] which in one dimension is [33] :

$$
\Delta T=2 T_{\mathrm{c}}^{0}\left(\frac{b T_{\mathrm{c}}^{0}(2 m)^{1 / 2}}{\alpha^{3 / 2}}\right)^{2 / 3} .
$$

In the clean case $(\chi=1)$ one has from the coefficients given above $\Delta T \approx 1.6 T_{\mathrm{c}}^{0}$ and even larger values are obtained for the dirty case which is probably realized in organic conductors. Thus, strong fluctuation effects are to be expected in a wide temperature range. Static properties of a strictly one-dimensional system like the specific heat and static correlation functions can be calculated exactly using functional integration methods [33]. However this method cannot be generalized to the dynamic properties like the conductivity. An approximate way to calculate the effect of the strong fluctuations is to use a Hartree-Fock-like procedure to account for the fourth-order term (3.9a). Then there appear "self-energy" corrections and the coefficient $\alpha$ has to be replaced by $\alpha+\Sigma$, where for the case of one-dimensional fluctuations $\left(\xi_{\perp}<a\right)$ the self-energy $\Sigma$ satisfies the self-consistency equation

$$
\Sigma=\frac{2 T b(2 m)^{1 / 2}}{(\alpha+\Sigma)^{1 / 2}} .
$$

This equation can be solved analytically. In figure 5 , we show the result for the square of the inverse coherence length, which is proportional to $\alpha+\Sigma$, together with the exact [33] and mean-field results for $\Delta T / T_{\mathrm{c}}^{0}=1$.

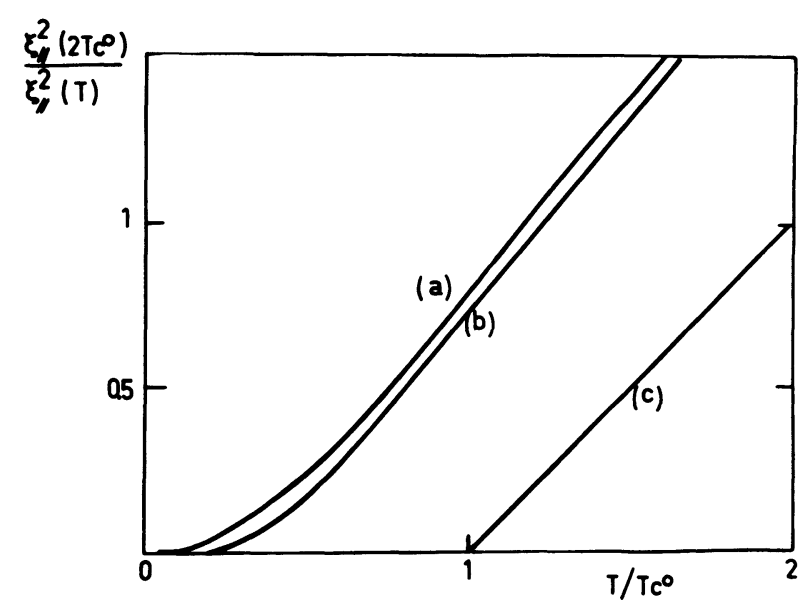

Fig. 5. - Temperature dependence of the inverse square of the correlation length of a one-dimensional system : (a) Hartree-Fock approximation, $(b)$ exact result, and $(c)$ mean-field theory result.

Here for simplicity $\alpha=\alpha^{\prime}\left(T / T_{\mathrm{c}}^{0}-1\right)$ and temperature independent constants $\alpha^{\prime}$ and $b$ have been assumed. The interesting feature of figure 5 is that the approximate solution is in very good agreement with the exact result down to nearly $T_{\mathrm{c}}^{0} / 2$. Above this temperature the solution is a nearly perfect straight line, which cuts the $T$-axis at a positive temperature. Therefore above about $T_{\mathrm{c}}^{0} / 2$, the temperature dependence of the fluctuation conductivity is given by equation (3.6), only $T_{\mathrm{c}}^{0}$ being replaced by some effective temperature $T_{0} \approx T_{\mathrm{c}}^{0} / 3$, see figure 5 .

For larger $\Delta T$ the temperature region where $\alpha+\Sigma$ is quasi-linear is even larger and $T_{0}$ correspondingly smaller. At very low temperatures the Hartree-Fock approximation fails. This is due to the fact that at these temperatures only the phase of the order parameter fluctuates, whereas the amplitude is nearly constant, in contrast to the situation at higher temperatures, where amplitude fluctuations are dominant due to the large value of $\Delta T$. The Hartree-Fock renormalization (3.11) describes the harmonic motion of the order parameter around the origin in an effective field, i.e. amplitude fluctuations. On the other hand, in the phase fluctuation region the amplitude is nearly fixed and only the phase of the order parameter rotates. Clearly, this cannot be described by a renormalized harmonic motion around the origin and therefore the Hartree-Fock approximation fails at very low temperatures. We remark, however, that the above description applies to one-dimensional fluctuations, i.e. for $\xi_{\perp}(T)<a$ in a quasi- 
one-dimensional conductor. At sufficiently low temperatures, however, one always has $\xi_{\perp}(T)>a$, and then the three-dimensional results (Eq. (3.6)) apply.

The Ginzburg-Landau calculation presented above gives, in microscopic terms, the contribution of the Azlamazov-Larkin (AL) diagram [35] to the fluctuation conductivity [27]. However, it cannot account for the Maki-Thompson diagrams [36, 37]. It is well known that these diagrams are very sensitive to pairbreaking effects and their contribution may thus be suppressed in the usually quite impure organic conductors. Inclusion of these diagrams and of the effect of the coupling between the BCS and the Peierls instability in one-dimensional conductors [38] requires a microscopic calculation. However we expect that the most interesting results, namely the strong enhancement of the fluctuation conductivity due to the large anisotropy (Eq. (3.6)) and the reduction of the fluctuation diamagnetism with respect to an isotropic superconductor (Eq. (3.10)) should be unchanged. A full account of the above presented calculations will be given in a subsequent publication [23].

\section{Comparison between theory and experiments. -}

We shall now attempt to see whether the large value and the temperature dependence of the conductivity of (TMTSF $)_{2} \mathrm{PF}_{6}$ can be related to the theory of paraconductivity in a very anisotropic conductor proposed in the previous section. For the purpose of comparison between theory and experimental data it is convenient to deal with the resistivity, which following (3.6a) becomes :

$$
\begin{aligned}
\rho_{\|}=\frac{8 \sqrt{2 m}}{\pi e^{\prime 2}} a^{2} \frac{\alpha^{3 / 2}}{\alpha^{\prime}} & \left(1+\frac{2 \pi J^{2}}{m v_{\mathrm{F}}^{2} \alpha}\right)^{1 / 2} \times \\
& \times\left(1+\left(1+\frac{2 \pi J^{2}}{m v_{\mathrm{F}}^{2} \alpha}\right)^{1 / 2}\right)
\end{aligned}
$$

with the notations of section 3 and :

$$
\alpha^{\prime}=\frac{4 k T \hbar}{\pi m v_{\mathrm{F}}^{2} \tau}
$$

where $\hbar / \tau$ is the energy broadening of the Fermi distribution at low temperature due the finite electron life time.

The limiting forms 1-D and 3-D for $\rho_{\|}$can be easily derived from $(4.1)$

$$
\begin{gathered}
\rho_{\|}^{3 \mathrm{D}}=5 \times 10^{-4}\left(\frac{T}{T_{\mathrm{F}}} \frac{\theta}{T_{\mathrm{F}}}\right)^{1 / 2} \frac{B}{T}\left(\ln \frac{T}{T_{\mathrm{c}}}\right)^{1 / 2} \\
\rho_{\|}^{1 \mathrm{D}}=10^{-3}\left(\frac{T}{T_{\mathrm{F}}} \frac{\theta}{T_{\mathrm{F}}}\right)^{1 / 2}\left(\ln \frac{T}{T_{0}}\right)^{3 / 2}(\Omega \mathrm{cm}) \\
\text { LE JOURNAL DE PHYSIQUE }- \text { T. 42, No } 7 \text {, JUILLET } 1981
\end{gathered}
$$

where the finite electron life time broadening of the Fermi distribution can be approximated by the optical scattering time measured in TTF-TCNQ at $100 \mathrm{~K}, \tau \sim 10^{-14} \mathrm{~s}$, and $a=4 \AA$.

$B$ in equation (4.2a) is related to the cross-over temperature between 1-D and 3-D regimes. According to equation (4.1) it is given by the relation $B=2 \pi^{2} t_{\perp}^{2} / \theta$. However, the latter derivation depends strongly on the approximate forms taken for the integrals in equation (4.1). So we prefer to use $B$ in the rest of this section as an adjustable parameter.

First, we pay attention to the $T$-domain very close to the superconducting transition at $T_{\mathrm{c}}=0.9 \mathrm{~K}$. Near $T_{\mathrm{c}}$ the transverse coherence length becomes larger than the interchain lattice spacing and the 3-D form of the resistivity, equation $(4.2 a)$, is expected to fit the data of figure 3 . The experimental value

$$
\rho(1 \mathrm{~K})=2 \times 10^{-6} \Omega \mathrm{cm}
$$

substituted into equation (4.2a), with $\theta / T_{\mathbf{F}}=0.2$, leads to $B=0.85 \mathrm{~K}$ and hence to a cross-over temperature $T^{*}=1.75 \mathrm{~K}$ with $T^{*}=T_{\mathrm{c}}+B$. Given the above derived value of $B$, we have plotted on figure $6 a$ the $T$-dependence of $\rho_{\|}^{3 \mathrm{D}}(T)$ up to $2 \mathrm{~K}$ (in a temperature domain where the 3-D approximate form for $\rho_{\|}$ is likely to be applicable).

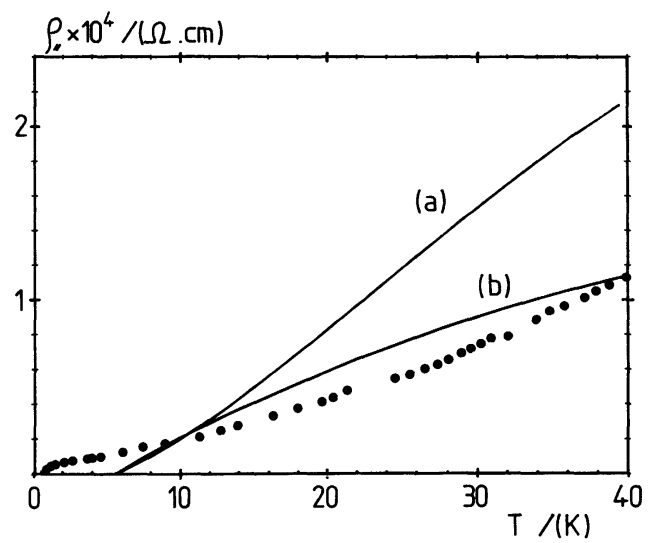

Fig. 6a. - Comparison of experimental resistivity (points) with the theoretical results (full lines) in the region of one-dimensional fluctuations : (a) with $T_{0}=5.6 \mathrm{~K}, \theta / T_{\mathrm{F}}=0.2$, and $a=3 \AA$ in equation $(4.2 b)$ and $(b)$ after introduction of a normal conductivity of $\sigma_{0}=4000(\Omega \mathrm{cm})^{-1}$, so that $\rho=\left(\sigma_{\|}^{1 \mathrm{D}}+\sigma_{0}\right)^{-1}$.

Secondly, the 1-D form of $\rho_{\|}$is expected to explain the temperature dependence of the resistivity as long as the paraconductivity of superconducting origin dominates over all other conductivity channels. This condition is likely to be fulfilled at helium temperature if paraconductivity is important. Thus the resistivity of the 1-D regime depends only on one additional parameter, $T_{0}$, the 1-D mean-field temperature. Given $T_{\mathrm{F}}=1000 \mathrm{~K}$ and $\theta / T_{\mathrm{F}}=0.2$ the experimental value at $10 \mathrm{~K}$, namely $\rho_{\|}^{1 \mathrm{D}}(10 \mathrm{~K})=2 \times 10^{-5} \mathrm{~cm}$ leads to $T_{0}=5.6 \mathrm{~K}$, according to equation $(4.2 b)$. At higher 
temperatures the $\rho_{\|}^{1 \mathrm{D}}(T)$ theoretical curve (with $\theta / T_{\mathrm{F}}=0.2, T_{0}=5.6 \mathrm{~K}$ ) deviates significantly from the observed data. For example at $40 \mathrm{~K}$, the theory leads to twice the observed resistivity, see figure $6 b$. The agreement between the theory and experimental data can be improved by realizing that the paraconductivity at $40 \mathrm{~K}$ calculated from equation $(4.2 b)$ amounts to $4166(\Omega \mathrm{cm})^{-1}$. This value is no longer large compared to other mechanisms of conduction, in particular the single-particle channel limited by impurities and (or) lattice imperfections. If we take a $T$-independent single particle contribution below $40 \mathrm{~K}$ equal to $4000(\Omega \mathrm{cm})^{-1}$ as observed in other organic conductors in absence of Fröhlich fluctuating conduction we obtain a theoretical resistivity

$$
\rho=\left(\sigma_{\|}^{1 \mathrm{D}}+\sigma_{0}\right)^{-1}
$$

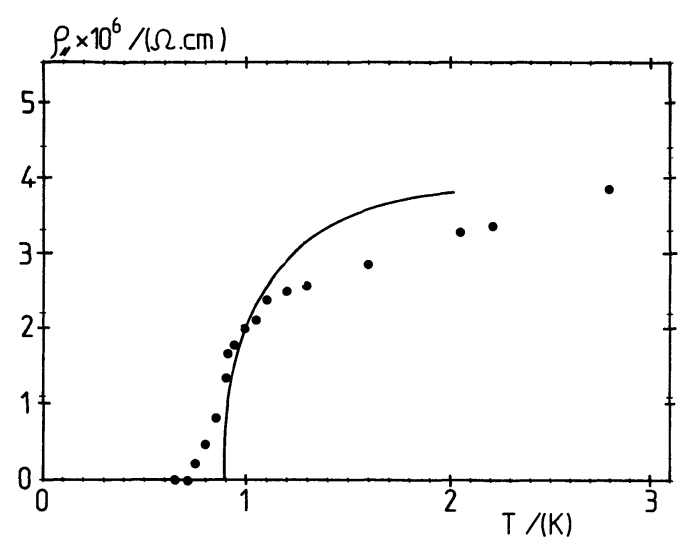

Fig. 6b. - Comparison of experimental resistivity (points) with the theoretical results (full line) in the region of three-dimensional fluctuations (Eq. (4.2a)) with $T_{\mathrm{c}}=0.9 \mathrm{~K}, \theta / T_{\mathrm{F}}=0.2$, and $B=0.85$.

which follows more closely the experimental data, see figure $6 b$. Moreover, the observed shape of the temperature dependence of $\rho_{\|}$between $40 \mathrm{~K}$ and $4.2 \mathrm{~K}$ can vary slightly from sample to sample. In some samples, we have noticed a steep descent of the resistivity below $40 \mathrm{~K}$, resembling closely the theoretical behaviour, equation $(4.2 b)$.

It is not unlikely that the sample of figure 7 is indeed more impure than that of figure 6 with a smaller contribution of the single-particle conduction channel at low temperature.

We wish to point out from the above interpretation that the low resistivity observed at low temperature in (TMTSF) ${ }_{2} \mathrm{PF}_{6}$ can be explained with very reasonable values of the band parameters within the frame work of the paraconductivity model, whereas a single particle model for the conduction would necessitate mean free paths of the order of $3000 \AA$ which seem unlikely in organic conductors.

In the 1-D regime of fluctuations, whenever the paraconduction is dominant over the single-particle conduction the anisotropy given by equation (3.9) is

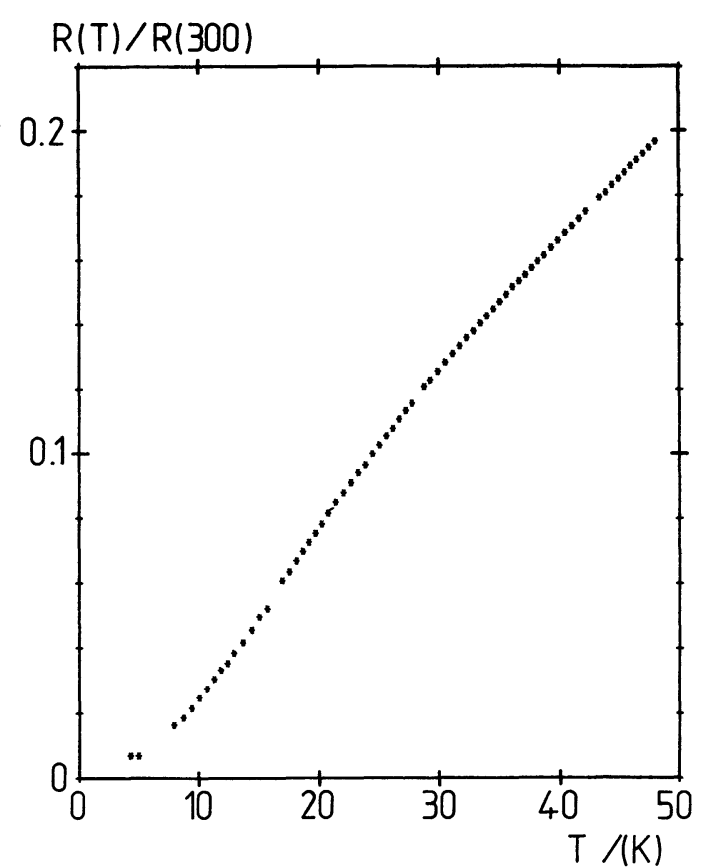

Fig. 7. - Temperature dependence of the longitudinal resistivity of another sample of (TMTSF) $)_{2} \mathrm{PF}_{6}$ at $12 \mathrm{kbar}$.

temperature dependent. In the vicinity of $T_{0}$ the anisotropy is given by :

$$
\sigma_{\|} / \sigma_{\perp} \approx\left(\frac{t_{\|}}{t_{\perp}}\right)^{2}\left(\frac{T-T_{0}}{T_{0}}\right)
$$

where $t_{\|}$and $t_{\perp}$ are the longitudinal and transverse electron transfer integrals, respectively.

Therefore, the decrease of the anisotropy towards low temperatures figure $2 a$ can be readily understood in terms of equation (4.3) and its value, order of magnitude wise, is similar to the value of the room temperature anisotropy $\approx\left(\frac{t_{\|}}{t_{\perp}}\right)^{2}$. Within the paraconductivity picture the anisotropy begins its low temperature descent when the paraconductive channel becomes a dominant process over the regular single-particle one. Figure $2 a$ shows that the onset temperature for the fluctuations is about $40 \mathrm{~K}$, in satisfying agreement with the quantitative analysis of the longitudinal conductivity.

Moreover an anisotropy of $10^{3}$ at high temperature dropping to about 300 at low temperature indicates a band structure anisotropy $t_{\|} / t_{\perp} \approx 30$, along the $\mathrm{Se}-\mathrm{Se}$ direction. As the temperature is further decreased towards the superconducting transition the anisotropy tends to saturate at a level given by equation (3.7) $\sigma_{\|} / \sigma_{\perp} \approx\left(t_{\|} / t_{\perp}\right)^{2}$. Such a value is also in good agreement with a study of the $H_{\mathrm{c}_{2}}$ critical field anisotropy presently under completion [39].

On figure 8 we have plotted the temperature dependence under $12 \mathrm{kbar}$ of the resistivity of the two-chain charge transfer complex TMTSF-DMTCNQ which can be stabilized in a conducting state at low temperature under pressure $[1,2]$. The conductivity of 


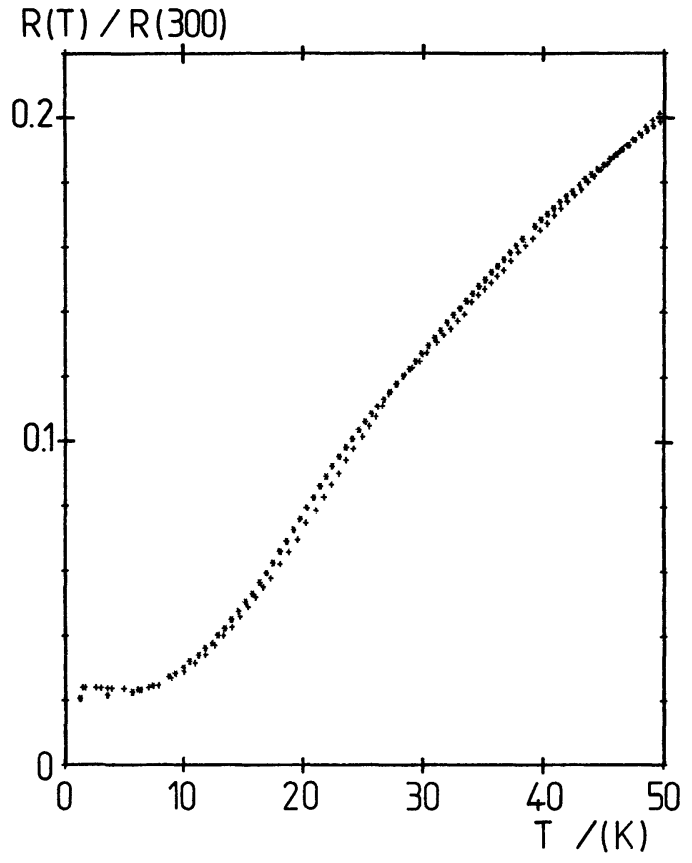

Fig. 8. - Temperature dependence of the longitudinal resistivity of two samples of TMTSF-DMTCNQ. At helium temperature and under a $12 \mathrm{kbar}$ pressure the conductivity reaches $10^{5}(\Omega \mathrm{cm})^{-1}[1,2]$.

TMTSF-DMTCNQ reaches $\approx 10^{5}(\Omega \mathrm{cm})^{-1}$ at helium temperature. However, contrary to the parent compound (TMTSF) ${ }_{2} \mathrm{PF}_{6}$ a saturation of the resistance is observed below $8 \mathrm{~K}$ and low temperaturehigh pressure studies down to $100 \mathrm{mK}$ have failed to detect superconductivity [40]. The reason for the absence of superconducting state in TMTSFDMTCNQ may lie in the presence of paramagnetic centres which have been found by susceptibility measurements [41] to be more abundant in TMTSFDMTCNQ than in (TMTSF) ${ }_{2} \mathrm{PF}_{6}$. In a quasi-onedimensional system impurities are expected to be far more effective in destroying long-range order than in an isotropic system due to the decoupling of the order parameters on adjacent chains [42]. Even if the long-range three-dimensional order is completely destroyed, however, there is still the possibility of large 1-D fluctuations on the individual chains. Therefore, despite significant differences between these two conductors as far as ordered state superconductivity is concerned we consider as very likely the existence of a strong paraconductive mechanism enhancing the conduction of TMTSF-DMTCNQ at low temperature [1]. The temperature dependence and amplitude of the conductivity are very similar in both compounds in the region of temperatures 40 to $10 \mathrm{~K}$ where paraconductivity could be the dominant conductivity channel in (TMTSF $)_{2} \mathrm{PF}_{6}$.

Attempts to detect strong fluctuation diamagnetism above $T_{\mathrm{c}}$ in (TMTSF) ${ }_{2} \mathrm{PF}_{6}$ have not given significant results, using a compensated coil AC technique in earth field [39].

Given the sensitivity of the experimental set-up, we find that the fluctuation induced diamagnetism is smaller than, say $10^{-4}$ times the diamagnetism corresponding to a Meissner effect, namely $1 / 4 \pi$. However these results are in agreement with the discussion of section 3 which has clearly established that the fluctuation diamagnetism above $T_{\mathrm{c}}$ is not enhanced in a quasi-one-dimensional conductors by dimensionality effects. Equation (3.10a) shows that in the low field perpendicular geometry the susceptibility in the 1-D regime becomes

$$
\chi_{\perp}^{\mathrm{DD}}=-\frac{k T}{6 \pi} \frac{e^{2}}{c^{2}} \xi_{\|}(T) \frac{2 \xi_{\perp}(T)}{a} .
$$

Compared to the value in an isotropic conductor, equation (4.4) indicates that $\chi$ is reduced by the ratio $\xi_{\perp}(T) / a<1$ in the $1-\mathrm{D}$ regime of fluctuations.

However there is still a possibility to observe significant diamagnetism in the temperature domain extending from $T_{\mathrm{c}}$ up to $T_{\mathrm{c}}^{0}$ where $\xi_{\|}(T)$ should retain a large value. This may be the interpretation for the rather large value of the diamagnetism found in TMTSF-DMTCNQ at low temperature under pressure [43]. We must notice that the theory of section 3 is a low-field theory, whereas measurements of the susceptibility in [43] were performed in a field of at least $20 \mathrm{kOe}$ or so. But it is not unlikely that the superconducting fluctuations survive the application of a rather large magnetic field since, due to the rather weak interchain coupling, the establishment of magnetic-field induced screening currents require large magnetic fields.

Finally, there is an other experimental result for (TMTSF $)_{2} \mathrm{PF}_{6}$ and TMTSF-DMTCNQ which could be understood with the suggestion of strong paraconductivity up to about $40 \mathrm{~K}$ : the unusually large effect of a magnetic field on the resistance (longitudinal or transverse) provided it is applied perpendicular to the chain direction $[1,2,7]$. Considering the relatively small transverse conductivity, magnetic fields much larger than the critical fields of the condensed superconducting state are necessary to destroy the fluctuations above $T_{\mathrm{c}}$. When the field is applied parallel to the conducting axis, the induced currents cannot take advantage of the large longitudinal conductivity and consequently the superconducting fluctuations are hardly affected by a field as large as $75 \mathrm{kOe}[1,2]$.

5. Nature of the ground state. - We now consider the microscopic origin of the superconducting instability in (TMTSF) ${ }_{2} \mathrm{PF}_{6}$. A one-dimensional interacting electron gas is usually described in terms of two coupling constants $g_{1}$ and $g_{2}$ for electron-electron backward and forward scattering respectively. Bychkov et al. [38] pointed out that the BCS and Peierls instabilities have to be considered simultaneously in one-dimension. Their results as well as renormalization group calculation $[44,45]$ indicate that for $g_{1}>0, g_{1}-2 g_{2}>0$ the system shows a BCS 
instability, for $g_{1}>0, g_{1}-2 g_{2}<0$ a Peierls (or spin density wave) instability, and for $g_{1}<0$ simultaneous BCS and Peierls instabilities. However, for $g_{1}<0$ the parquet [38] and renormalization $[44,45]$ methods are unreliable and mean-field $[46,49]$ theory as well as scaling $[50,51]$ to an exactly solvable case [52] show that for $g_{1}<0$ the line $g_{1}=2 g_{2}$ separates the regions of BCS and Peierls instabilities. In these calculations electron-phonon interaction may be included by an effective coupling constant and an energy cut off of the order of the phonon frequency. If the retardation of the electron-phonon interaction is fully accounted for, it has been shown $[46,49]$ that for strong coupling the Peierls state is stable even in the region $g_{1}<0, g_{1}-2 g_{2}>0$.

In a strictly one-dimensional system an ordered state may only occur at $T=0$. In quasi-one-dimensional systems however, there are always interactions between neighbouring chains, leading to phase transitions at finite temperature. The Coulomb interactions between neighbouring chains couple charge density waves [53] and therefore leads to a Peierls transition at finite temperature [54, 57]. On the other hand, coupling between superconducting order parameters requires electrons to move from one chain to the next, so that a BCS transition may only occur for finite interchain tunnelling integral $[55,56$, 59].

To apply these results to an organic superconductor like (TMTSF) ${ }_{2} \mathrm{PF}_{6}$ we note first that the interchain tunnelling coupling is weak compared to the coupling of the charge density waves due to the small interchain coupling integral. This can be seen by noting that the anisotropy of the Peierls coherence length in a typical organic conductor like TTF-TCNQ [60] is about 10 whereas the anisotropy of the superconducting coherence length, equation (3.3), is proportional to the ratio of longitudinal and transverse tight-binding integrals, which is of the order 30 in typical organic conductors $[9,26]$. This is in agreement with the large anisotropy of the conductivity of (TMTSF) $)_{2} \mathrm{PF}_{6}$ [6]. Therefore, to obtain the observed superconducting transition with $g_{1}<0$, i.e. attractive interactions, one has to be well on the left of the $g_{1}=2 g_{2}$, line since near to this line, even for $g_{1}-2 g_{2}>0$ there is still a weak divergence of the Peierls response function which due to the relatively large interchain Coulomb coupling would lead to a Peierls transition. In fact, preliminary X-ray measurements [61], indicate no CDW fluctuations above the Peierls-transition at ambient pressure, in contrast to other 1-D conductors [60]. An effectively attractive interaction is obtained by electron-phonon interactions [64-66]. Acoustic phonons are not expected to give a significant contribution, as their cut off, namely, the Debye frequency, is very small compared to the Fermi energy, which is the cut off for the electron-electron Coulomb interaction. On the other hand intramolecular modes have often very high frequencies, and, as pointed out by Horovitz [48], high frequencies are favourable for a high BCS transition temperature. In fact, it is well known that in organic conductors intramolecular modes of high frequencies may be strongly coupled to the conduction electrons [63-66].

A possible explanation of the superconductivity instability is therefore an intramolecular-phonon mediated electron-electron attraction. This would bring confirmation to the early ideas of Little [67] on the possible role of intramolecular interactions, though of a somewhat different kind, in organic superconductivity.

Another possible explanation, due to Barisic and Brazovskii [68] would be a strong reduction of the long range $\left(g_{2}\right)$ part of the Coulomb interaction due to non-logarithmic screening effects. This would allow to reach the $g_{1}>2 g_{2}$ region even for $g_{1}$, $g_{2}>0$, i.e. there would be a superconducting instability with repulsive interactions.

6. Conclusion. - The present study was devoted towards understanding the unusual transport properties of (TMTSF) ${ }_{2} \mathrm{PF}_{6}$ at $12 \mathrm{kbar}$ above $T_{\mathrm{s}}$, namely, the longitudinal conductivity in excess of $10^{5}(\Omega \mathrm{cm})^{-1}$ at low temperature and the ratio $\sigma_{\|} / \sigma_{\perp}$ decreasing below $50 \mathrm{~K}$. We do not find satisfactory the interpretation of the conduction in terms of a singleparticle model with a transverse conduction becoming coherent at low temperature since this would mean extremely large longitudinal mean free paths of about $3000 \AA$. Instead, we claim that a fluctuation-enhanced conduction of superconducting origin can explain the experimental data. We have presented the calculations of the effects of Azlamazov-Larkin diagrams on the onset of superconducting above the phase transition in the frame work of the time dependent GinzburgLandau theory. The theory provides two different regimes for the paraconductivity (i) a 3-D regime restricted to the very vicinity of $T_{\mathrm{c}}$ namely $\frac{\Delta T_{\mathrm{c}}}{T_{\mathrm{c}}} \lesssim 1$ where $\xi_{\perp} / a>1$ and $\sigma_{\|}^{3 \mathrm{D}} \sim T^{1 / 2}\left(\ln T / T_{\mathrm{c}}\right)^{-1 / 2}$ and (ii) a 1-D regime, with $\xi_{\perp} / a<1$ and $\sigma_{\|}^{1 \mathrm{D}} \sim T^{-1 / 2}\left(\ln T / T_{0}\right)^{-3 / 2}$, where $T_{0}$ represents some kind of renormalized mean-field temperature, which can very well be depressed from the bare mean-field temperature $T_{\mathrm{c}}^{0}$ by a factor 3 or so.

We wish to point out that the proposed theory for paraconductivity leads to reasonable agreement with the experimental data. Around $40 \mathrm{~K}$, the paraconductive contribution is already equal to the single-particle contribution and it is about 25 times larger than the single-particle conductivity at low temperature.

The latter remark supports the point of view [69] that observation at low temperature of conductivity of the order of that of copper in 1-D organic conductors involves superconductivity. Although, one-dimensionality enhances drastically the paraconductivity with respect to its value in a regular isotropic super- 
conductor (by a factor $(\xi(0) / a)^{2} \approx 10^{5}$ in $(\mathrm{TMTSF})_{2} \mathrm{PF}_{6}$ at $T=2 T_{\mathrm{c}}$ ) the fluctuation diamagnetism is not enhanced in the 1-D temperature regime. This latter point is in agreement with the failure to detect strong diamagnetic fluctuations in $(\mathrm{TMTSF})_{2} \mathrm{PF}_{6}$.
Acknowledgments. - We wish to acknowledge the help of our colleagues at Orsay and Copenhagen who participated in the experimental efforts. We have also benefited from discussion with $\mathbf{J}$. Friedel on several theoretical aspect of this work.

\section{References}

[1] Andrieux, A., Duroure, C., Jérome, D. and BechgaArd, K. J. Physique Lett. 40 (1979) L-381.

[2] Andrieux, A., Chaikin, P. M., Duroure, C., Jérome, D., Weyl, C., Bechgaard, K. and Andersen, J. R., J. Physique 40 (1979) 1199.

[3] Friend, R. H., Miljak, M., Jérome, D., Decker, D. L. and Debray, D., J. Physique Lett. 39 (1978) L-134.

[4] Etemad, S., Phys. Rev. B 13 (1976) 2254.

[5] Cooper, J. R., Weger, M., Jérome, D., Lefur, D., BechgaArd, K., Bloch, A. N. and Cowan, D. O., Solid State Commun. 19 (1976) 749.

[6] Bechgaard, K., Jacobsen, C. S., Mortensen, K., PederSEN, H. J. and Thorup, N., Solid State Commun. 33 (1980) 1119.

[7] Jérome, D., Mazaud, A., Ribault, M. and BechgaArd, K., J. Physique Lett. 41 (1980) L-95.

[8] Ribault, M., Benedek, G., Jérome, D. and BechgaArd, K. J. Physique Lett. 41 (1980) L-397.

[9] Soda, G., Jérome, D., Weger, M., Alizon, J., Gallice, J., Robert, H., Fabre, J. M. and Giral, L., J. Physique 38 (1977) 931.

[10] Cooper, J. R., Jérome, D., Etemad, S. and Engler, E. M., Solid State Commun. 22 (1977) 257.

[11] ANDrieux, A., Schulz, H. J., Jérome, D. and BeChGaArd, K., J. Physique Le't. 40 (1979) L-385.

[12] JÉrome, D., Proceedings of the NATO ASI Conference in Tomar Portugal 1979, L. Alcacer editor (D. Reidel Publishing Company) 1980.

[13] Jérome, D. and Schulz, H. J., Extended Linear Chain Compounds, J. S. Miller, editor (Plenum publishing Corporation), in press.

[14] Jérome, D. and Weger, M., in Chemistry and Physics of One Dimensional Metals (Plenum, New York) 1977.

[15] Mott, N. F. and DAvis, E. A., Electronic Processes in NonCrystalline Materials (Clarendon Press, Oxford) 1971.

[16] Recent X-ray and transport properties investigations have shown that the crystals used in reference [5] were imperfect. In good quality-crystals a rather sharp transition is observed at $24 \mathrm{~K}$ at ambient pressure both by transport and X-ray scattering experiments. This transition is not suppressed by high pressure up to $24 \mathrm{kbar}$ at least. (Orsay work to be published.)

[17] Cooper, J. R., Weger, M., Delplanque, G., Jérome, D., Bechgaard, K., J. Physique Lett. 37 (1976) L-349.

[18] Weger, M., Solid State Commun. 19 (1976) 1149.

[19] Gorkov, L. P., Sov. Phys. J.E.T.P. 9 (1959) 1364 ; Sov. Phys. J.E.T.P. 10 (1960) 998.

[20] DE Gennes, P. G., Superconductivity of Metals and Alloys (Benjamin, New York) 1966.

[21] Lawrence, W. F. and Doniach, S., in Proceedings of the 12th International Conference on Low Temperature Physics, edited by E. Kandu (Academic Press of Japan, Tokyo) 1971 , p. 361.

[22] Horovitz, B., Gutfreund, H. and Weger, M., Phys. Rev. B 12 (1975) 3174

[23] Schulz, H. J., to be published.

[24] Tilley, D. R., Proc. Phys. Soc. 86 (1965) 289.

[25] Morris, R. C., Coleman, R. V. and Bhandari, R., Phys. Rev. B 5 (1972) 895
[26] Berlinsky, A. J., Carolan, J. F. and Weiler, L., Solid State Commun. 15 (1974) 795

[27] Schmidt, H., Z. Phys. 216 (1968) 336

[28] Schmm, A., Phys. Kondens. Mater. 5 (1966) 302.

[29] Abrahams, E. and Tsuneto, T., Phys. Rev. 152 (1966) 416.

[30] Turkevich, L. A. and Klemm, R. A., Phys. Rev. B 19 (1979) 2520.

[31] Schmid, A., Phys. Rev. 180 (1969) 527.

[32] Ginzburg, V. L., Sov. Phys. Solid. State 2 (1960) 1824.

[33] Scalapino, D. J., Sears, M. and Ferrell, R. A., Phys. Rev. B 6 (1972) 3409.

[34] Schmid, A., Z. Phys. 229 (1969) 81.

[35] Azlamazov, L. G. and Larkin, A. T., Sov. Phys. Solid State 10 (1968) 875.

[36] Maki, K., Progr. Theor. Phys. 40 (1968) 193.

[37] Thompson, R. S., Phys. Rev. B 1 (1970) 327.

[38] Bychkov, Yu. A., Gorkov, L. P., DzyaloshinskiI, I. E., Sov. Phys. J.E.T.P. 23 (1966) 489.

[39] Orsay Work, Ribault, M., Mailly, D., Jérome, D. and BECHGAARD, K., to be published.

[40] Orsay WORK, Bouffard, S., Ribault, M., to be published.

[41] Private communication from J. R. COOPER to the authors.

[42] Abrahams, E., Gorkov, L. P., Kharadze, G. A., J. Low. Temp. Phys. 23 (1978) 673.

Bergman, D. J., Rice, T. M. and Lee, P. A., Phys. Rev. B 15 (1977) 1706.

[43] Hardebusch, V., Gerhardt, W., Schilling, J. S., BechGAARD, K., Weger, M., MilJaK, M. and COOPER, J. R., Solid State Commun. 32 (1979) 1151.

[44] Menyhard, N. and Solyom, J., J. Low Temp. Phys. 12 (1973) 529.

[45] 'Solyom, J., J. Low Temp. Phys. 12 (1973) 547.

[46] Gutfreund, H., Horovitz, B. and Weger, M., J. Phys. C 7 (1974) 383.

[47] Levin, K., Mills, D. L. and Cunningham, S. L., Phys. Rev. $B 10$ (1974) 3821.

[48] Horovitz, B., Solid State Commun. 18 (1976) 445

[49] Horovitz, B., Phys. Rev. B 16 (1977) 3843.

[50] LeE, P. A., Phys. Rev. Lett. 34 (1975) 1247.

[51] Emery, V. J., Luther, A. and Peschel, I., Phys. Rev. B 13 (1976) 1272.

[52] Luther, A. and Emmery, V. J., Phys. Rev. Lett. 33 (1974) 589.

[53] Saub, K., Barišic, S. and Friedel, J., Phys. Lett. 56A (1976) 302.

[54] Gorkov, L. P. and DzyaloshinskiI, I. E., Sov. Phys. J.E.T.P. 40 (1975) 198.

[55] Klemm, R. A. and Gutfreund, H., Phys. Rev. B 14 (1976) 1086 , and

Gutfreund, H. and Klemm, R. A., Phys. Rev. B 14 (1976) 1073.

[56] Minaly, L. and Solyom, J., J. Low Temp. Phys. 24 (1976) 579.

[57] Lee, P. A., Rice, T. M. and Klemm, R. A., Phys. Rev. B 15 (1977) 2984.

[58] Prigodin, V. N. and Firsov, Yu. A., Sov. Phys. J.E.T.P. 49 (1979) 813.

[59] Suzumura, Y. and Fukuyama, H., J. Low Temp. Phys. 31 (1978), 273.

[60] Khanna, S. K. et al., Phys. Rev. B 16 (1977) 1468.

[61] Pouget, J. P., private communication and to be published. 
[62] Barišić, S., J. Physique Colloq. 39 (1978) C2-263.

[63] Gorkov, L. P., in Lecture Notes in Physics, n $^{\circ} 96$ (Springer Verlag) 1979.

[64] Rice, M. J., Duke, C. B. and LiPARI, N. O., Solid State Commun. 17 (1975) 1089.

[65] Rice, M. J. and Lipari, N. O., Phys. Rev. Lett. 38 (1977) 337.

[66] Horovitz, B., Gutfreund, H. and Weger, M., Phys. Rev. B 17 (1978) 2796.
[67] Little, W. A., Phys. Rev. 134 (1964) A 1416 and Gutfreund, H. and Little, W. A., in Chemistry and Physics of One-dimensional Metals, H. J. Keller, editor (Plenum Press) 1977.

[68] Barišić, S. and BrazovskiI, S., Proc. EPS Conference, Antwerpen, 1980.

[69] Litrle, W. A., in Chemistry and Physics of One-dimensional Metals, H. J. Keller, editor (Plenum Press) 1977. 